\title{
Superintegrability on the two-dimensional hyperboloid
}

\author{
E. G. Kalnins \\ Department of Mathematics and Statistics, University of Waikato, Hamilton, New Zealand
}

W. Miller, Jr.

School of Mathematics, University of Minnesota, Minneapolis, Minnesota 55455

G. S. Pogosyan

Laboratory of Theoretical Physics, Joint Institute for Nuclear Research,

Dubna, Moscow Region 141980, Russia

(Received 19 May 1997; accepted for publication 30 June 1997)

\begin{abstract}
In this work we examine the basis functions for classical and quantum mechanical systems on the two-dimensional hyperboloid that admit separation of variables in at least two coordinate systems. We present all of these cases from a unified point of view. In particular, all of the special functions that arise via variable separation have their essential features expressed in terms of their zeros. The principal new results are the details of the polynomial bases for each of the nonsubgroup bases, not just the subgroup spherical coordinate cases, and the details of the structure of the quadratic symmetry algebras. (c) 1997 American Institute of Physics. [S0022-2488(97)03610-4]
\end{abstract}

\section{INTRODUCTION}

In a previous article ${ }^{1}$ we have studied the so-called superintegrable Hamiltonian systems in two-dimensional Euclidean space and the two-dimensional sphere. In that article we showed how to compute the basis functions for all the bound state wave functions for all the possible coordinate systems for which separation was possible. We recall that the notion of a superintegrable system relates to a potential for which the solution via separation of variables is possible in more than one coordinate system. ${ }^{2-4}$ In two dimensions there remains the case of the two-dimensional hyperboloid. Here we investigate this case, making suitable use of what we already know from Refs. 5, 6 and earlier papers such as Refs. 7-16. The main emphasis will be on those features that are new, together with the role that the quadratic algebra has in these computations. There are four potentials listed in Ref. 6 that permit multiple variable separation on the hyperboloid, but we limit our attention to the first three, since the remaining potential does not give rise to bound states. (In distinction to the case for Euclidean space and the sphere, a complete listing of distinct superintegrable systems on the two-dimensional hyperboloid has not yet been achieved. For some additional superintegrable systems, see Ref. 5.) We study each of the three cases systematically. We will consider two examples of expansions relating bases associated to different coordinate systems. In the first example we use the structure of the quadratic algebra relations to compute an expansion; in the second we compute directly.

For each of the superintegrable systems we observe that, for the discrete spectrum of the quantum mechanical Hamiltonian, one can consider this operator as acting on a space of polynomials in appropriate variables. ${ }^{17}$ Each eigenvalue is multiply degenerate. However, every separable coordinate system gives rise to an orthonormal basis of polynomial eigenfunctions in this space and breaks the degeneracy. These bases are characterized as simultaneous eigenfunctions of second-order symmetry operators for the Hamiltonian. ${ }^{18}$ We show that under commutation the symmetry operators close to form a quadratic algebra, ${ }^{11}$ and we determine the structure of that algebra. The superintegral systems are of two types: the "normal type" in which the original Hamiltonian is diagonalized, and the "conformal type" in which the Hamiltonian is modified by multiplying the eigenvalue equation by a function and considering the energy as fixed. The 
TABLE I. Separable coordinates for three superintegrable systems.

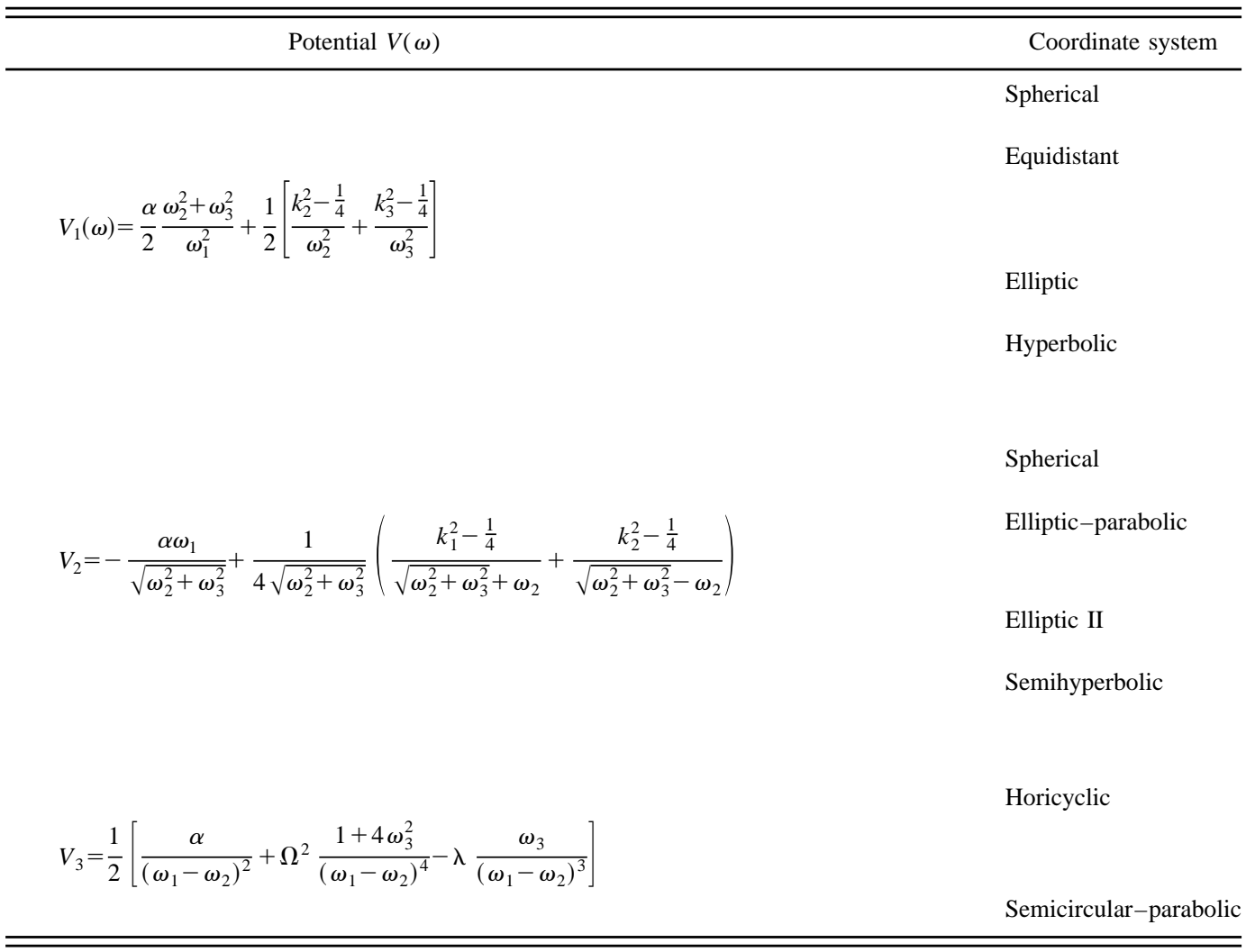

modified equation is then interpreted as the eigenvalue equation for a Hamiltonian on a conformal Euclidean space with a "charge" as the eigenvalue.

\section{SOLUTIONS OF THE SCHRÖDINGER EQUATION}

The two-dimensional hyperboloid is characterized via the Cartesian coordinates $\omega_{1}, \omega_{2}, \omega_{3}$, where $\omega_{1}^{2}-\omega_{2}^{2}-\omega_{3}^{2}=1, \omega_{1}>1$. The notion $\omega_{1}>1$ means that we consider only one sheet of the double-sheet hyperboloid. Throughout this paper we will consider the Schrödinger equation on the hyperboloid in the form $(\hbar=m=1)$

$$
H \Psi \equiv\left(-\frac{1}{2} \Delta_{\mathrm{LB}}+V\right) \Psi=E \Psi,
$$

where $V$ is the potential function and the Laplace-Beltrami operator $\Delta_{\mathrm{LB}}$ can be written as

$$
\Delta_{\mathrm{LB}}=K_{3}^{2}+K_{2}^{2}-M_{1}^{2}
$$

Here $K_{3}, K_{2}, M_{1}$ generate the Lie algebra $\operatorname{so}(2,1),{ }^{18}$

$$
K_{3}=w_{1} \partial_{w_{2}}+w_{2} \partial_{w_{1}}, \quad K_{2}=w_{1} \partial_{w_{3}}+w_{3} \partial_{w_{1}}, \quad M_{1}=\omega_{2} \partial_{\omega_{3}}-\omega_{3} \partial_{\omega_{2}}
$$

We now consider the potentials $V$ for which (1) is superintegrable, see Table I. 


\section{A. First potential}

The first potential considered in the paper ${ }^{6}$ is the singular oscillator potential,

$$
V_{1}=\frac{\alpha}{2} \frac{\omega_{2}^{2}+\omega_{3}^{2}}{\omega_{1}^{2}}+\frac{1}{2}\left[\frac{k_{2}^{2}-\frac{1}{4}}{\omega_{2}^{2}}+\frac{k_{3}^{2}-\frac{1}{4}}{\omega_{3}^{2}}\right],
$$

where $\alpha$ and $k_{2,3}$ are constants with $\alpha>0$, and $k_{2,3}>0$. The corresponding Schrödinger equation admits separable solutions in four coordinate systems: spherical, equidistant, elliptic, and hyperbolic. Unlike the corresponding oscillator system on the two-dimensional sphere and Euclidean space, the potential (4) on the hyperboloid has a discrete and a continuous spectrum. The bound state solutions can be found as follows.

\section{Spherical coordinates}

Here,

$$
\omega_{1}=\cosh \tau, \quad \omega_{2}=\sinh \tau \cos \varphi, \quad \omega_{3}=\sinh \tau \sin \varphi
$$

$(\tau>0, \varphi \in[0,2 \pi))$ and the potential (4) reads as

$$
V_{1}(\tau, \varphi)=\frac{\alpha}{2}+\frac{1}{2}\left[\frac{1}{\sinh ^{2} \tau}\left(\frac{k_{2}^{2}-\frac{1}{4}}{\cos ^{2} \varphi}+\frac{k_{3}^{2}-\frac{1}{4}}{\sin ^{2} \varphi}\right)-\frac{k_{1}^{2}-\frac{1}{4}}{\cosh ^{2} \tau}\right],
$$

where $k_{1}=\sqrt{\alpha+\frac{1}{4}}$. Choosing the wave function $\Psi$ in the form

$$
\Psi_{1}(\tau, \varphi)=(\sinh \tau)^{-1 / 2} S(\tau) \Phi(\varphi),
$$

we go to the system of coupled differential equations in the Pöschl-Teller form

$$
\begin{gathered}
\frac{d^{2} S}{d \tau^{2}}+\left[\widetilde{E}+\frac{k_{1}^{2}-\frac{1}{4}}{\cosh ^{2} \tau}-\frac{\lambda^{2}-\frac{1}{4}}{\sinh ^{2} \tau}\right] S=0, \\
\frac{d^{2} \Phi}{d \varphi^{2}}+\left[\lambda^{2}-\left(\frac{k_{2}^{2}-\frac{1}{4}}{\cos ^{2} \varphi}+\frac{k_{3}^{2}-\frac{1}{4}}{\sin ^{2} \varphi}\right)\right] \Phi=0,
\end{gathered}
$$

where $\widetilde{E}=2 E+\alpha-\frac{1}{4}$ and $\lambda$ is a spherical separation constant. The solution of the equation (9) normalized in the region $\varphi \in[0, \pi / 2]$ has the form ${ }^{2}$

$$
\begin{aligned}
\Phi(\varphi)=\Phi_{m}^{\left( \pm k_{3}, \pm k_{2}\right)}(\varphi)= & \sqrt{\frac{2\left(2 m+1 \pm k_{2} \pm k_{3}\right) m ! \Gamma\left(1+m \pm k_{2}+k_{3}\right)}{\Gamma\left(1+m \pm k_{2}\right) \Gamma\left(1+m \pm k_{3}\right)}} \\
& \times(\sin \varphi)^{1 / 2 \pm k_{3}}(\cos \varphi)^{1 / 2 \pm k_{2}} P_{m}^{\left( \pm k_{3}, \pm k_{2}\right)}(\cos 2 \varphi),
\end{aligned}
$$

with $m \in \mathbf{N}$, where $P_{n}^{(\alpha, \beta)}$ is the Jacobi polynomial. ${ }^{19}$ The separation constant is quantized as

$$
\lambda=\left(2 m \pm k_{2} \pm k_{3}+1\right) .
$$

The positive sign at $k_{2,3}$ has to be taken if $k_{2,3}>1 / 2$ and both positive and negative signs must be taken if $0<k_{2,3}<1 / 2$, so the function $\Phi$ has a finite form.

The second (modified) Pöschl-Teller equation (8) was investigated by Frank and Wolf ${ }^{20}$ and Barut $e t a l .{ }^{21}$ The bound state wave function is given by 


$$
\begin{aligned}
S(\tau) \equiv S_{n}^{\left(\lambda, k_{1}\right)}(\tau) & =N_{n}^{\left(\lambda, k_{1}\right)}(\sinh \tau)^{1 / 2+\lambda}(\cosh \lambda)^{2 n-k_{1}+1 / 2}{ }_{2} F_{1}\left(-n, k_{1}-n ; 1+\lambda ; \tanh ^{2} \tau\right) \\
& =\frac{n ! \Gamma(1+\lambda)}{\Gamma(1+\lambda+n)} N_{n}^{\left(\lambda, k_{1}\right)}(\sinh \tau)^{1 / 2+\lambda}(\cosh \tau)^{1 / 2-k_{1}} P_{n}^{\left(\lambda,-k_{1}\right)}(\cosh 2 \tau)
\end{aligned}
$$

with $n=0,1, \ldots\left[\frac{1}{2}\left(k_{1}-\lambda-1\right)\right]$ and a bound state solution is possible only for $k_{1}-\lambda>1$. The wave function $S_{n}^{\left(\lambda, k_{1}\right)}(\tau)$ satisfies the orthogonality relation

$$
\int_{0}^{\infty} S_{n}^{\left(\lambda, k_{1}\right)}(\tau) S_{n^{\prime}}^{\left(\lambda, k_{1}\right)}(\tau) d \tau=\delta_{n n^{\prime}}
$$

and the normalization factor has the form

$$
N_{n}^{\left(\lambda, k_{1}\right)}=\frac{1}{\Gamma(1+\lambda)} \sqrt{\frac{2\left(k_{1}-\lambda-2 n-1\right) \Gamma(1+\lambda+n) \Gamma\left(k_{1}-n\right)}{\Gamma\left(k_{1}-\lambda-n\right) n !}} .
$$

Here, and in the remainder of this paper, the bound state wave functions are normalized so that they form an orthonormal set with respect to the invariant measure on the hyperboloid (which in spherical coordinates is $\sinh \tau d \tau d \varphi$ ).

The normalized total wave function $\Psi_{1}(\tau, \varphi) \equiv \Psi_{n m}\left(\tau, \varphi ; k_{1}, \pm k_{2}, \pm k_{3}\right)$ is given by (7), (10), (12), and (13). The quantized energy is

$$
E_{N}=-\frac{1}{2}\left[\left(2 N-k_{1} \pm k_{2} \pm k_{3}+2\right)^{2}-\frac{1}{4}\right]+\frac{\alpha}{2},
$$

where $N=m+n$ is the principal quantum number and the bound states occur for

$$
0 \leqslant N \leqslant \frac{1}{2}\left(k_{1} \mp k_{2} \mp k_{3}-2\right) .
$$

Each solution $\Psi_{1}(\tau, \varphi)$ satisfies the eigenvalue equation

$$
L_{1} \Psi_{1} \equiv\left[M_{1}^{2}-\left(\omega_{2}^{2}+\omega_{3}^{2}\right)\left(\frac{k_{2}^{2}-\frac{1}{4}}{\omega_{2}^{2}}+\frac{k_{3}^{2}-\frac{1}{4}}{\omega_{3}^{2}}\right)\right] \Psi_{1}=-4\left(m+\frac{1 \pm k_{2} \pm k_{3}}{2}\right)^{2} \Psi_{1},
$$

and $L_{1}$ commutes with $H$.

\section{Equidistant coordinates}

Here,

$\omega_{1} \cosh a \cosh b, \quad \omega_{2}=\cosh a \sinh b, \quad \omega_{3}=\sinh a$

$\left[\tau_{1,2} \in(-\infty, \infty)\right]$ and the potential $V_{1}$ has the form

$$
V_{1}(a, b)=\frac{\alpha}{2}-\frac{1}{2}\left[\frac{1}{\cosh ^{2} a}\left(\frac{k_{1}^{2}-\frac{1}{4}}{\cosh ^{2} b}-\frac{k_{2}^{2}-\frac{1}{4}}{\sinh ^{2} b}\right)-\frac{k_{3}^{2}-\frac{1}{4}}{\sinh ^{2} a}\right] .
$$

After putting

$$
\Psi_{2}(a, b)=(\cosh a)^{-1 / 2} S(a) S(b),
$$

we go to the two modified Pöschl-Teller equations, 


$$
\begin{gathered}
\frac{d^{2} S}{d a^{2}}+\left[\widetilde{E}+\frac{\mu^{2}-\frac{1}{4}}{\cosh ^{2} a}-\frac{k_{3}^{2}-\frac{1}{4}}{\sinh ^{2} a}\right] S=0, \\
\frac{d^{2} S}{d b^{2}}+\left[-\mu^{2}+\left(\frac{k_{1}^{2}-\frac{1}{4}}{\cosh ^{2} b}-\frac{k_{2}^{2}-\frac{1}{4}}{\sinh ^{2} b}\right)\right] S=0,
\end{gathered}
$$

where $\mu$ is the equidistant separation constant. The normalized for $a, b \in[0, \infty)$ bound state wave functions have the form

$$
\begin{aligned}
S(b) \equiv S_{n_{1}}^{\left( \pm k_{2}, k_{1}\right)}(b)= & \sqrt{\frac{2\left(k_{1} \mp k_{2}-1-2 n_{1}\right) \Gamma\left(k_{1}-n_{1}\right) n_{1} !}{\Gamma\left(k_{1} \mp k_{2}-n_{1}\right) \Gamma\left(1 \pm k_{2}+n_{1}\right)}} \\
& \times(\sinh b)^{1 / 2 \pm k_{2}}(\cosh b)^{1 / 2-k_{1}} P_{n_{1}}^{\left( \pm k_{2},-k_{1}\right)}(\cosh 2 b) \\
S(a) \equiv S_{n_{2}}^{\left( \pm k_{3}, \mu\right)}(a)= & \sqrt{\frac{2\left(\mu \mp k_{3}-1-2 n_{2}\right) \Gamma\left(\mu-n_{2}\right) n_{2} !}{\Gamma\left(\mu \mp k_{3}-n_{2}\right) \Gamma\left(1 \pm k_{3}+n_{2}\right)}} \\
& \times(\sinh a)^{1 / 2 \pm k_{3}}(\cosh a)^{1 / 2-\mu} P_{n_{2}}^{\left( \pm k_{3},-\mu\right)}(\cosh 2 a)
\end{aligned}
$$

and the separation constant is

$$
\mu=\left(k_{1} \mp k_{2}-2 n_{1}\right),
$$

with $n_{1}=0,1, \ldots\left[\frac{1}{2}\left(k_{1} \mp k_{2}-1\right)\right]$ and $n_{2}=0,1, \ldots\left[\frac{1}{2}\left(\mu \mp k_{3}-1\right)\right]$. The bound state energy $E_{N}$ is given by (14), where now the principal quantum number $N$ is $N=n_{1}+n_{2}$ and satisfies the equation (15). The total wave function $\Psi_{2}(a, b) \equiv \Psi_{n_{1} n_{2}}\left(a, b ; k_{1}, \pm k_{2}, \pm k_{3}\right)$ satisfies

$$
L_{3} \Psi_{2} \equiv\left[-K_{3}^{2}-\left(\omega_{1}^{2}-\omega_{2}^{2}\right)\left(\frac{k_{1}^{2}-\frac{1}{4}}{\omega_{1}^{2}}-\frac{k_{2}^{2}-\frac{1}{4}}{\omega_{2}^{2}}\right)\right] \Psi_{2}=-4\left(n_{1}+\frac{1-k_{1} \pm k_{2}}{2}\right)^{2} \Psi_{2},
$$

where $\left[L_{3}, H\right]=0$.

We can introduce also the second equidistant system of coordinates by the interchange $\omega_{2} \leftrightarrow \omega_{3}$. Then the total bound state wave function $\Psi_{3}(\widetilde{a}, \widetilde{b}) \equiv \Psi_{\widetilde{n}_{1}, \widetilde{n_{2}}}\left(\widetilde{a}, \widetilde{b} ; k_{1}, \pm k_{2}, \pm k_{3}\right)$ may be obtained from (20) and (21) by the interchange $k_{2} \leftrightarrow k_{3}$ and satisfies

$$
L_{2} \Psi_{3}=\left[-K_{2}^{2}-\left(\omega_{1}^{2}-\omega_{3}^{2}\right)\left(\frac{k_{1}^{2}-\frac{1}{4}}{\omega_{1}^{2}}-\frac{k_{3}^{2}-\frac{1}{4}}{\omega_{3}^{2}}\right)\right] \Psi_{3}=-\left(\widetilde{n_{1}}+\frac{1-k_{1} \pm k_{3}}{2}\right)^{2} \Psi_{3},
$$

where $\left[L_{2}, H\right]=0$.

We can call these three bases that are the eigenfunctions of the Hamiltonian for the potential $V_{1}$ and of each of the operators $\left(L_{1}, L_{2}, L_{3}\right)$ the fundamental bases for the potential of a singular oscillator on the two-dimensional hyperboloid. Note also that

$$
L_{1}+L_{2}+L_{3}=2 H-\left(2 k_{1}^{2}+k_{2}^{2}+k_{3}^{2}-1\right)
$$




\section{Elliptic coordinates of type one}

For the remaining elliptic-type coordinates it is convenient to introduce the quantities

$$
s_{1}^{2}=\frac{\left(\mu-e_{j}\right)\left(\nu-e_{j}\right)}{\left(e_{j}-e_{k}\right)\left(e_{j}-e_{l}\right)}, \quad i, j, k=1,2,3, \quad \text { and } \quad \neq
$$

For elliptic coordinates of type one, the coordinates on the hyperboloid are given by

$$
\omega_{1}^{2}=s_{1}^{2}, \quad \omega_{2}^{2}=-s_{2}^{2}, \quad \omega_{3}^{2}=-s_{3}^{2},
$$

where $e_{1}<e_{2}<\nu<e_{3}<\mu$. Rather than express the wave functions in the separable coordinates $\mu$, $\nu$, we will use a Niven equations approach, ${ }^{22}$ in which the separated solutions are expressed in Cartesian coordinates and the critical information is the location of the zeros of these solutions. The bound state wave functions are

$$
\Psi_{4}=\left(\Pi_{l=1}^{3} \omega_{l}^{k_{l}+1 / 2}\right) \Pi_{j=1}^{q}\left[\frac{\omega_{1}^{2}}{\theta_{j}-e_{1}}-\frac{\omega_{2}^{2}}{\theta_{j}-e_{2}}-\frac{\omega_{3}^{2}}{\theta_{j}-e_{3}}\right],
$$

where the $\theta_{j}$ satisfy

$$
\frac{k_{1}+1}{\theta_{j}-e_{1}}+\frac{k_{2}+1}{\theta_{j}-e_{2}}+\frac{k_{3}+1}{\theta_{j}-e_{3}}+\sum_{j \neq m} \frac{2}{\theta_{m}-\theta_{j}}=0
$$

These solutions satisfy the eigenvalue equations

$$
\begin{aligned}
\left(e_{3} L_{3}+e_{2} L_{2}+e_{1} L_{1}\right) \Psi_{4}= & \left(\sum_{i, j, l \neq}\left[-2 k_{i}\left(e_{j}+e_{l}\right)+e_{i} k_{j} k_{l}-4 e_{i} e_{j}\left(k_{l}+1\right) \sum_{m=1}^{q} \frac{1}{\theta_{m}-e_{l}}\right]\right. \\
& \left.-\frac{3}{2}\left(e_{1}+e_{2}+e_{3}\right)\right) \Psi_{4} .
\end{aligned}
$$

\section{Elliptic coordinates of type two}

The coordinates on the hyperboloid are given by

$$
\omega_{1}^{2}=s_{2}^{2}, \quad \omega_{2}^{2}=-s_{1}^{2}, \quad \omega_{3}^{2}=-s_{3}^{2},
$$

where $\nu<e_{1}<e_{2}<e_{3}<\mu$. The bound state wave functions are

$$
\Psi_{5}=\left(\Pi_{l=1}^{3} \omega_{l}^{e_{l}+1 / 2}\right) \Pi_{j=1}^{q}\left[-\frac{\omega_{2}^{2}}{\theta_{j}-e_{1}}+\frac{\omega_{1}^{2}}{\theta_{j}-e_{2}}-\frac{\omega_{3}^{2}}{\theta_{j}-e_{3}}\right],
$$

where $\epsilon_{1}=k_{2}, \epsilon_{2}=k_{1}, \epsilon_{3}=k_{3}$. The $\theta_{j}$ satisfy

$$
\frac{\epsilon_{1}+1}{\theta_{j}-e_{1}}+\frac{\epsilon_{2}+1}{\theta_{j}-e_{2}}+\frac{\epsilon_{3}+1}{\theta_{j}-e_{3}}+\sum_{j \neq m} \frac{2}{\theta_{m}-\theta_{j}}=0 .
$$

The eigenvalue equation is 


$$
\begin{aligned}
\left(e_{3} L_{3}+e_{2} L_{1}+e_{1} L_{2}\right) \Psi_{5}= & \left(\sum_{i, j, l \neq}\left[-2 \epsilon_{i}\left(e_{j}+e_{l}\right)+e_{i} \epsilon_{j} \epsilon_{l}-4 e_{i} e_{j}\left(\epsilon_{l}+1\right) \sum_{m=1}^{q} \frac{1}{\theta_{m}-e_{l}}\right]\right. \\
& -\frac{3}{2}\left(e_{1}+e_{2}+e_{3}\right) \Psi_{5} .
\end{aligned}
$$

\section{Quadratic algebra relations}

Here, we define the operator $R$ by the equality

$$
R=\left[L_{1}, L_{2}\right]=\left[L_{3}, L_{1}\right]=\left[L_{2}, L_{3}\right],
$$

and can verify that $R$ satisfies

$$
\left[L_{j}, R\right]=-4\left\{L_{j}, L_{m}-L_{l}\right\}+8\left[\left(\frac{3}{2}-k_{m}^{2}\right) L_{m}-\left(\frac{3}{2}-k_{l}^{2}\right) L_{l}\right]+8\left(k_{l}^{2}-k_{m}^{2}\right),
$$

where $j, m, l$ is an even permutation of $1,2,3$. In addition,

$$
\begin{aligned}
R^{2}= & \frac{8}{3}\left\{L_{1}, L_{2}, L_{3}\right\}-16\left(1-k_{1}^{2}\right) L_{1}^{2}-16\left(1-k_{2}^{2}\right) L_{2}^{2}-16\left(1-k_{3}^{2}\right) L_{3}^{2}+\frac{52}{3}\left(\left\{L_{1}, L_{2}\right\}+\left\{L_{2}, L_{3}\right\}\right. \\
& \left.+\left\{L_{1}, L_{3}\right\}\right)+\frac{1}{3}\left(\left(60-176 k_{1}^{2}\right) L_{1}+\left(60-176 k_{2}^{2}\right) L_{2}+\left(60-176 k_{3}^{2}\right) L_{3}\right)+64 k_{1} k_{2} k_{3} \\
& +48\left(k_{1} k_{2}+k_{2} k_{3}+k_{1} k_{3}\right)+\frac{32}{3}\left(k_{1}+k_{2}+k_{3}\right),
\end{aligned}
$$

where $\{A, B\}=A B+B A$ and

$$
\{A, B, C\}=A B C+A C B+B C A+B A C+C A B+C B A .
$$

\section{B. Second potential}

The singular Coloumb-Kepler potential is

$$
V_{2}=-\frac{\alpha \omega_{1}}{\sqrt{\omega_{2}^{2}+\omega_{3}^{2}}}+\frac{1}{4 \sqrt{\omega_{2}^{2}+\omega_{3}^{2}}}\left(\frac{k_{1}^{2}-\frac{1}{4}}{\sqrt{\omega_{2}^{2}+\omega_{3}^{2}}+\omega_{2}}+\frac{k_{2}^{2}-\frac{1}{4}}{\sqrt{\omega_{2}^{2}+\omega_{3}^{2}}-\omega_{2}}\right),
$$

where $\alpha>0$ and $k_{1,2}>0$. The Schrödinger equation for this potential can be solved for the bound state wave functions in four coordinate systems: spherical, elliptic-parabolic, elliptic II, semihyperbolic.

\section{Spherical coordinates}

In coordinates (5) we have

$$
V_{2}(\tau, \varphi)=-\alpha \operatorname{coth} \tau+\frac{1}{8 \sinh ^{2} \tau}\left(\frac{k_{1}^{2}-\frac{1}{4}}{\cos ^{2}(\varphi / 2)}+\frac{k_{2}^{2}-\frac{1}{4}}{\sin ^{2}(\varphi / 2)}\right) .
$$

Choosing the wave functions in form,

$$
\Psi_{1}=(\sinh \tau)^{-1 / 2} S(\tau) \Phi_{m}^{\left( \pm k_{2}, \pm k_{1}\right)}(\varphi / 2),
$$

where the wave function $\Phi_{m}^{\left( \pm k_{2}, \pm k_{1}\right)}(\varphi / 2)$ normalized for $\varphi \in[0, \pi]$ is given by (10) and $m$ $\in \mathbf{N}$. After separation of variables we come to the equation 


$$
\frac{d^{2} S}{d \tau^{2}}+\left[\widetilde{E}+2 \alpha \operatorname{coth} \tau-\frac{\lambda^{2}-\frac{1}{4}}{\sinh ^{2} \tau}\right] S=0,
$$

where $\widetilde{E}=2 E-\frac{1}{4}$. This equation was first introduced by Manning and Rosen ${ }^{23}$ to study the vibrations of diatomic molecules.

For the bound state wave function normalized in region $\tau \in[0, \infty)$, we have

$$
\begin{aligned}
S(\tau) \equiv & S_{n m}(\tau) \\
= & \frac{2^{m+1 \pm k_{1} / 2 \pm k_{2} / 2}}{\Gamma\left(2 m+2 \pm k_{1}+k_{2}\right)}\left[\frac{\left(\sigma_{N}^{2}-N^{2}\right)}{N} \frac{\Gamma\left(n+2 m+2 \pm k_{1} \pm k_{2}\right)}{n !} \frac{\Gamma\left(\sigma_{N}+m+1 \pm k_{1} / 2 \pm k_{2} / 2\right)}{\Gamma\left(\sigma_{N}-m \mp k_{1} / 2 \mp k_{2} / 2\right)}\right]^{1 / 2} \\
& \times(\sinh \tau)^{m+1 \pm k_{1} / 2 \pm k_{2} / 2} e^{-\tau\left(\sigma_{N}-n\right)} \\
& \times{ }_{2} F_{1}\left(-n, m+1 \pm \frac{k_{1}}{2} \pm \frac{k_{2}}{2}+\sigma_{N} ; 2 m+2 \pm k_{1} \pm k_{2} ; \frac{2}{1+\operatorname{coth} \tau}\right),
\end{aligned}
$$

where $\sigma_{N}=\alpha / N$ and $N=n+m+1 \pm k_{1} / 2 \pm k_{2} / 2$. The quantisation condition for the energy is

$$
E_{N}=-\frac{N^{2}-\frac{1}{4}}{2}-\frac{\alpha^{2}}{2 N^{2}}
$$

and the bound states occur for

$$
0 \leqslant n+m \leqslant\left[\sqrt{\alpha} \mp \frac{k_{1}}{2} \mp \frac{k_{2}}{2}-1\right] .
$$

The solutions $\Psi_{1} \equiv \Psi_{n m}\left(\tau, \varphi ; \pm k_{1} \pm k_{2}\right)$ are eigenfunctions of the symmetry operator

$$
L=M_{1}^{2}-\frac{1}{2} \sqrt{\omega_{2}^{2}+\omega_{3}^{2}}\left(\frac{k_{1}^{2}-\frac{1}{4}}{\sqrt{\omega_{2}^{2}+\omega_{3}^{2}}+\omega_{2}}+\frac{k_{2}^{2}-\frac{1}{4}}{\sqrt{\omega_{2}^{2}+\omega_{3}^{2}}-\omega_{2}}\right),
$$

with eigenvalue $-\left[m+1 / 2\left(1 \pm k_{1} \pm k_{2}\right)\right]^{2}$.

\section{Semihyperbolic coordinates}

Here

$$
\omega_{1}=\frac{1+x y}{\sqrt{\left(y^{2}-1\right)\left(x^{2}-1\right)}}, \quad \omega_{2}=\frac{y-x}{\sqrt{\left(y^{2}-1\right)\left(x^{2}-1\right)}}, \quad \omega_{3}=\frac{2 \sqrt{x y}}{\sqrt{\left(y^{2}-1\right)\left(x^{2}-1\right)}},
$$

where $x, y \in[0,1)$. [Note that these coordinates are connected with corresponding coordinates in Ref. 6 by the transformation $\mu_{1}=2 x /\left(x^{2}-1\right)$ and $\left.\mu_{2}=2 y /\left(y^{2}-1\right)\right]$. The potential $V_{2}$ now has the form

$$
V_{2}(x, y)=-\alpha \frac{x y+1}{x+y}+\frac{1}{8} \frac{\left(x^{2}-1\right)\left(y^{2}-1\right)}{x+y}\left(\frac{k_{1}^{2}-\frac{1}{4}}{y}+\frac{k_{2}^{2}-\frac{1}{4}}{x}\right) .
$$

The computation of the bound state wave functions proceeds as follows. The Schrödinger equation has the form 


$$
\begin{aligned}
& \sqrt{x\left(x^{2}-1\right)} \frac{\partial}{\partial x} \sqrt{x\left(x^{2}-1\right)} \frac{\partial \Psi}{\partial x}+\sqrt{y\left(y^{2}-1\right)} \frac{\partial}{\partial y} \sqrt{y\left(y^{2}-1\right)} \frac{\partial \Psi}{\partial y}+\left[2 E\left(\frac{x}{x^{2}-1}+\frac{y}{y^{2}-1}\right)\right. \\
& \left.+\alpha\left(\frac{x^{2}+1}{x^{2}-1}+\frac{y^{2}+1}{y^{2}-1}\right)-\frac{x y-1}{4}\left(\frac{k_{1}^{2}-\frac{1}{4}}{y}+\frac{k_{2}^{2}-\frac{1}{4}}{x}\right)\right] \Psi=0 .
\end{aligned}
$$

Choosing the wave function in the form $\Psi_{2}(x, y)=X(x) Y(y)$, after the separation of variables we have two equations:

$$
\begin{aligned}
& x\left(x^{2}-1\right)\left[\frac{d^{2} X}{d x^{2}}+\frac{1}{2}\left(\frac{1}{x}+\frac{1}{x-1}+\frac{1}{x+1}\right) \frac{d X}{d x}\right] \\
& +\left[\frac{1}{2}(2 \alpha+\lambda)+\frac{2 \alpha}{x^{2}-1}-\frac{1}{4}\left(k_{1}^{2}-\frac{1}{4}\right) x+\frac{k_{2}^{2}-\frac{1}{4}}{4 x}+\frac{2 E x}{x^{2}-1}\right] X=0, \\
& y\left(y^{2}-1\right)\left[\frac{d^{2} Y}{d y^{2}}+\frac{1}{2}\left(\frac{1}{y}+\frac{1}{y-1}+\frac{1}{y+1}\right) \frac{d Y}{d y}\right] \\
& +\left[\frac{1}{2}(2 \alpha-\lambda)+\frac{2 \alpha}{y^{2}-1}-\frac{1}{4}\left(k_{2}^{2}-\frac{1}{4}\right) y+\frac{k_{1}^{2}-\frac{1}{4}}{4 y}+\frac{2 E y}{y^{2}-1}\right] Y=0,
\end{aligned}
$$

where $\lambda$ is a semihyperbolic separation constant. The first of these equations can be solved as follows. Looking for solutions of the form

$$
\begin{aligned}
X= & x^{(1 / 2)\left(1 / 2 \pm k_{2}\right)}(x-1)^{-(1 / 4)\left(2 p+1 \pm k_{1} \pm k_{2}\right)+\alpha\left(2 p+2 \pm k_{1} \pm k_{2}\right)^{-1}} \\
& \times(x+1)^{-(1 / 4)\left(2 p+1 \pm k_{1} \pm k_{2}\right)-\alpha\left(2 p+2 \pm k_{1} \pm k_{2}\right)^{-1}} \Pi_{j=1}^{p}\left(x-\xi_{1}\right),
\end{aligned}
$$

we see that the zeros $\xi_{i}$ satisfy

$$
\frac{1 \pm k_{2}}{\xi_{i}}+\left(1 \pm k_{1}\right) \xi_{i}-2 \sum_{j \neq i} \frac{\xi_{i} \xi_{j}-1}{\xi_{i}-\xi_{j}}-\frac{2 \alpha}{p+1 \pm k_{1} / 2 \pm k_{2} / 2}=0, \quad i=1, \ldots, p .
$$

A similar solution for the equation in $y$ can be obtained via the substitution

$$
\begin{aligned}
Y= & y^{(1 / 2)\left(1 / 2 \pm k_{1}\right)}(y-1)^{-(1 / 4)\left(2 p+1 \pm k_{1} \pm k_{2}\right)+\alpha\left(2 p+2 \pm k_{1} \pm k_{2}\right)^{-1}} \\
& \times(y+1)^{-(1 / 4)\left(2 p+1 \pm k_{1} \pm k_{2}\right)-\alpha\left(2 p+2 \pm k_{1}+k_{2}\right)^{-1}} \prod_{j=1}^{p}\left(y-\zeta_{i}\right) .
\end{aligned}
$$

The zeros satisfy

$$
\frac{1 \pm k_{1}}{\zeta_{i}}+\left(1 \pm k_{2}\right) \zeta_{i}-2 \sum_{j \neq i} \frac{\zeta_{i} \zeta_{j}-1}{\zeta_{i}-\zeta_{j}}-\frac{2 \alpha}{p+1 \pm k_{1} / 2 \pm k_{2} / 2}=0, \quad i=i, \ldots, p
$$

The separation constant $\lambda$ has the value 


$$
\begin{aligned}
\lambda & =2\left(1 \pm k_{1}\right)\left(\xi_{1}+\cdots+\xi_{p}\right)-4 \alpha \frac{p \pm k_{2} / 2 \mp k_{1} / 2}{2 p+2 \pm k_{1} \pm k_{2}} \\
& =-2\left(1 \pm k_{2}\right)\left(\zeta_{1}+\cdots+\zeta_{p}\right)+4 \alpha \frac{p \pm k_{1} / 2 \mp k_{2} / 2}{2 p+2 \pm k_{1} \pm k_{2}}
\end{aligned}
$$

The equality of these two expressions can be seen by noting that the equations for the zeros can be obtained from each other via the substitutions $k_{1} \leftrightarrow k_{2}, \xi_{i} \leftrightarrow 1 / \zeta_{i}$. The symmetry operator with eigenvalue $\lambda$ is

$$
M=2 A_{2}+\frac{\omega_{1} \omega_{3}^{2}}{2 \sqrt{\omega_{2}^{2}+\omega_{3}^{2}}}\left[\frac{k_{1}^{2}-\frac{1}{4}}{\left(\sqrt{\omega_{2}^{2}+\omega_{3}^{2}}+\omega_{2}\right)^{2}}-\frac{k_{2}^{2}-\frac{1}{4}}{\left(\sqrt{\omega_{2}^{2}+\omega_{3}^{2}}-\omega_{2}\right)^{2}}\right],
$$

where $A_{2}$ is a component of the two-dimensional Runge-Lenz vector, ${ }^{24}$

$$
A_{i}=\frac{1}{2}\left\{K_{i}, M_{1}\right\}+\frac{\alpha \omega_{i}}{\sqrt{\omega_{2}^{2}+\omega_{3}^{2}}}, \quad i=2,3
$$

\section{Elliptic parabolic coordinates}

Here we have

$$
\omega_{1}=\frac{\cosh ^{2} a+\cos ^{2} \theta}{2 \cosh a \cos \theta}, \quad \omega_{2}=\frac{\sinh ^{2} a-\sin ^{2} \theta}{2 \cosh a \cos \theta}, \quad \omega_{3}=\tanh a \tan \theta,
$$

where $a>0, \theta \in(-\pi / 2, \pi / 2)$. The potential in these coordinates has the form

$$
V_{2}(a, \theta)=-\alpha \frac{\cosh ^{2} a+\cos ^{2} \theta}{\cosh ^{2} a-\cos ^{2} \theta}+\frac{1}{2} \frac{\cosh ^{2} a \cos ^{2} \theta}{\cosh ^{2} a-\cos ^{2} \theta}\left(\frac{k_{1}^{2}-\frac{1}{4}}{\sinh ^{2} a}+\frac{k_{2}^{2}-\frac{1}{4}}{\sin ^{2} \theta}\right) .
$$

Putting for the wave function $\Psi_{3}(a, \theta)=S(a) \Phi(\theta)$, after separation of variables we get two equations in Pöschl-Teller form:

$$
\begin{aligned}
& \frac{d^{2} S}{d a^{2}}+\left[-\mu^{2}+\frac{\nu^{2}-\frac{1}{4}}{\cosh ^{2} a}-\frac{k_{1}^{2}-\frac{1}{4}}{\sinh ^{2} a}\right] S=0, \\
& \frac{d^{2} \Phi}{d \theta^{2}}+\left[\mu^{2}-\left(\frac{\beta^{2}-\frac{1}{4}}{\cos ^{2} \theta}+\frac{k_{2}^{2}-\frac{1}{4}}{\sin ^{2} \theta}\right)\right] \Phi=0,
\end{aligned}
$$

where $\mu$ is a elliptic parabolic separation constant and

$$
\beta=\sqrt{\frac{1}{4}-2(E+\alpha)}, \quad \nu=\sqrt{\frac{1}{4}-2(E-\alpha)} .
$$

The separation constant is quantized as

$$
\mu=\left(2 n_{1}+\beta \pm k_{2}+1\right)=\left(\nu \mp k_{1}-1-2 n_{2}\right),
$$

and introducing the quantum number $N=n_{1}+n_{2}+1 \pm k_{1} / 2 \pm k_{2} / 2$ we go to the energy quantization (36) and condition (37) for $n_{1}+n_{2}$.

The total bound state wave function $\Psi_{3}(a, \theta) \equiv \Psi_{n_{1} n_{2}}(a, \theta)$ satisfies the orthogonality relation 


$$
\int_{0}^{\infty} \int_{0}^{\pi / 2} \Psi_{n_{1} n_{2}}(a, \theta) \Psi_{n_{1}^{\prime} n_{2}^{\prime}}(a, \theta) \frac{\cosh ^{2} a-\cos ^{2} \theta}{\cosh ^{2} a \cos ^{2} \theta} d a d \theta=\delta_{n_{1} n_{1}^{\prime}} \delta_{n_{2}, n_{2}^{\prime}}
$$

and has the form

$$
\Psi_{n_{1} n_{2}}(a, \theta)=\sqrt{\frac{\sigma_{N}^{2}-N^{2}}{\left(2 \sigma_{N}+2 n_{1}-2 n_{2} \pm k_{2} \mp k_{1}\right) N}} S_{n_{2}}^{\left( \pm k_{1}, \nu\right)}(a) \Phi_{n_{1}}^{\left( \pm k_{2}, \beta\right)}(\theta),
$$

where the wave function $\Phi_{n_{1}}^{\left( \pm k_{2}, \beta\right)}(\theta)$ is given by (10) and

$$
\begin{aligned}
S_{n_{2}}^{\left( \pm k_{1}, \nu\right)}(a)= & \sqrt{\frac{2\left(\nu \overline{+} k_{1}-1-2 n_{2}\right) \Gamma\left(\nu-n_{2}\right) n_{2} !}{\Gamma\left(\nu \overline{+} k_{1}-n_{2}\right) \Gamma\left(1 \pm k_{1}+n_{2}\right)}}(\sinh a)^{1 / 2 \pm k_{1}}(\cosh a)^{1 / 2-\nu} P_{n_{2}}^{\left( \pm k_{1},-\nu\right)} \\
& \times(\cosh 2 a) .
\end{aligned}
$$

The elliptic parabolic wave function (49) also satisfies the operator equation,

$$
\Lambda \Psi_{n_{1}, n_{2}}=\left(2 L-M-2 H-\frac{k_{1}^{2}+k_{2}^{2}-\frac{1}{2}}{2}\right) \Psi_{n_{1} n_{2}}=\left(\sigma_{N}+n_{1}-n_{2} \pm \frac{k_{2}}{2} \mp \frac{k_{1}}{2}\right)^{2} \Psi_{n_{1} n_{2}} .
$$

\section{Modified elliptic coordinate systems}

$$
\begin{gathered}
\omega_{1}=\cosh f \sqrt{\frac{\left(\rho_{1}-a_{3}\right)\left(\rho_{2}-a_{3}\right)}{\left(a_{3}-a_{2}\right)\left(a_{3}-a_{1}\right)}}+\sinh f \sqrt{-\frac{\left(\rho_{1}-a_{2}\right)\left(\rho_{2}-a_{2}\right)}{\left(a_{2}-a_{1}\right)\left(a_{2}-a_{3}\right)}}, \\
\omega_{2}=\sinh f \sqrt{\frac{\left(\rho_{1}-a_{3}\right)\left(\rho_{2}-a_{3}\right)}{\left(a_{3}-a_{2}\right)\left(a_{3}-a_{1}\right)}+\cosh f \sqrt{-\frac{\left(\rho_{1}-a_{2}\right)\left(\rho_{2}-a_{2}\right)}{\left(a_{2}-a_{1}\right)\left(a_{2}-a_{3}\right)}}} \\
\omega_{3}=\sqrt{-\frac{\left(\rho_{1}-a_{1}\right)\left(\rho_{2}-a_{1}\right)}{\left(a_{1}-a_{2}\right)\left(a_{1}-a_{3}\right)}}
\end{gathered}
$$

where

$$
\sinh f=\sqrt{\frac{a_{1}-a_{2}}{a_{2}-a_{3}}}, \quad \cosh f=\sqrt{\frac{a_{1}-a_{3}}{a_{2}-a_{3}}},
$$

and $a_{3}<a_{2}<\rho_{2}<a_{1}<\rho_{1}$. The solution for the bound state wave functions is facilitated by making the change of variables,

$$
\begin{gathered}
a_{2}=a_{1}+\frac{1}{4}\left(A_{+}+A_{-}\right)^{2}, \quad a_{3}=a_{1}+\frac{1}{4}\left(A_{+}-A_{-}\right)^{2}, \\
\rho_{j}=a_{1}+\frac{1}{4}\left(A_{+}^{2}+A_{-}^{2}\right)+\frac{1}{4} A_{+} A_{-}\left(R_{j}+\frac{1}{R_{j}}\right), \quad j=1,2 .
\end{gathered}
$$

In terms of these coordinates, the coordinates on the hyperboloid are given by 


$$
\begin{aligned}
& \omega_{1}=-\frac{R_{1} R_{2}+1}{2 \sqrt{R_{1} R_{2}}}, \quad \omega_{2}=-\frac{\left(A_{+}^{2}+A_{-}^{2}\right)\left(R_{1} R_{2}+1\right)+2 A_{+} A_{-}\left(R_{1}+R_{2}\right)}{2\left(A_{+}^{2}-A_{-}^{2}\right) \sqrt{R_{1} R_{2}}}, \\
& \omega_{3}=\frac{i \sqrt{\left(A_{+} R_{1}+A_{-}\right)\left(A_{+} R_{2}+A_{-}\right)\left(A_{-} R_{1}+A_{+}\right)\left(A_{-} R_{2}+A_{+}\right)}}{\left(A_{+}^{2}-A_{-}^{2}\right) \sqrt{R_{1} R_{2}}} .
\end{aligned}
$$

The corresponding bound state solutions are

$$
\Psi_{4}=\left(\Pi_{l=1}^{3} U_{l}^{k_{l}+1 / 2}\right) \Pi_{j=1}^{g}\left(\frac{U_{1}^{2}}{\theta_{j}+\Lambda_{-}}+\frac{U_{2}^{2}}{\theta_{j}+\Lambda_{+}}+\frac{U_{3}^{2}}{\theta_{j}}\right),
$$

where

$$
U_{1}^{2}=\frac{\left(R_{1}+\Lambda_{-}\right)\left(R_{2}+\Lambda_{-}\right)}{\Lambda_{-}^{2}-1}, \quad U_{2}^{2}=\frac{\left(R_{1}+\Lambda_{+}\right)\left(R_{2}+\Lambda_{+}\right)}{\Lambda_{+}^{2}-1}, \quad U_{3}^{2}=R_{1} R_{2},
$$

and $\Lambda_{+}=A_{+} / A_{-}, \Lambda_{-}=A_{-} / A_{+}$. The zeros $\theta_{j}$ satisfy

$$
\frac{k_{1}+1}{\theta_{m}+\Lambda_{-}}+\frac{k_{2}+1}{\theta_{m}+\Lambda_{+}}+\frac{\sqrt{\frac{1}{4}+2(E-\alpha)}+1}{\theta_{m}}+\sum_{j \neq m} \frac{2}{\theta_{m}-\theta_{j}}=0 .
$$

The symmetry operator characterizing this coordinate system is

$$
L^{\prime}=\left(\Lambda_{+}+\Lambda_{-}\right)(2 L-H)-2\left(\Lambda_{+}-\Lambda_{-}\right) M,
$$

with eigenvalues

$$
\begin{aligned}
\mu= & 2\left[\Lambda_{-}\left(k_{2}+\sqrt{\frac{1}{4}+2(E-\alpha)}\right)+\Lambda_{+}\left(k_{1}+\sqrt{\frac{1}{4}+2(E-\alpha)}\right)+\left[\Lambda_{+} k_{1}+\Lambda_{-} k_{2}\right]\right. \\
& \left.\times \sqrt{\frac{1}{4}+2(E-\alpha)}\right]+\frac{3}{2}\left(\Lambda_{+}+\Lambda_{-}\right)+\sqrt{\frac{1}{4}+2(E-\alpha)}\left[4 \Lambda_{+}\left(k_{1}+1\right)\right. \\
& \left.\times \sum_{m=1}^{n} \frac{1}{\theta_{m}+\Lambda_{-}}+4 \Lambda_{+}\left(k_{2}+1\right) \sum_{m=1}^{n} \frac{1}{\theta_{m}+\Lambda_{+}}\right]-4 \sqrt{\frac{1}{4}+2(E-\alpha)} \sum_{m=1}^{n} \frac{1}{\theta_{m}} .
\end{aligned}
$$

\section{Quadratic algebra relations}

The commutation relations for the quadratic algebra with basis $L, M$, and $H$ are, for $R$ $=[L, M]$,

$$
\begin{gathered}
{[R, M]=-24 L^{2}+3 M^{2}+16 H L+\left(10-4\left(k_{1}^{2}+k_{2}^{2}\right)\right) L+\left(k_{2}^{2}-k_{1}^{2}\right) M} \\
-\left(1+2\left(k_{1}^{2}+k_{2}^{2}\right)\right) H+\frac{1}{2}\left(k_{1}^{2}+k_{2}^{2}\right)-\frac{1}{4}-8 \alpha^{2}, \\
{[R, L]=-2\{L, M\}+\left(k_{1}^{2}-k_{2}^{2}\right) L+M+\frac{1}{2}\left(\frac{1}{4}+\alpha\right)\left(k_{1}^{2}-k_{2}^{2}\right),} \\
R^{2}=-16 H^{2} L+16 L^{3}+16 L^{2} H-\frac{2}{3}\{M, M, L\}+8 H\{L, M\}+8\left(k_{1}^{2}+k_{2}^{2}-6\right) L^{2}-\frac{11}{3} M^{2}-\frac{44}{3} H^{2} \\
-\frac{44}{3} H L+\frac{44}{3} M L+\left(2\left(k_{1}^{2}+k_{2}^{2}\right)-1\right)\{L, M\}+\left(\frac{41}{12}-\frac{19}{3}\left(k_{1}^{2}+k_{2}^{2}\right)+4 \alpha^{2}-4 k_{1}^{2} k_{2}^{2}\right) L \\
\left.+\left(\frac{10}{3}-8\left(k_{1}^{2}+k_{2}^{2}\right)+\left(k_{1}^{2}-k_{2}^{2}\right)\left(k_{1}^{2}-k_{2}^{2}\right)+4 \alpha^{2}\right)\right) H+\left(-\frac{11}{6}+\frac{11}{3}\left(k_{1}^{2}+k_{2}^{2}\right)\right.
\end{gathered}
$$




$$
\begin{aligned}
& \left.+\frac{88}{3} \alpha\left(k_{2}^{2}-k_{1}^{2}\right)\right) M+\alpha^{2}\left(2\left(k_{1}^{2}+k_{2}^{2}\right)-\frac{1}{3}\right)+\frac{1}{2}\left(k_{1}^{2}-k_{2}^{2}\right)\left(2\left(k_{1}^{2}+k_{2}^{2}\right)-1\right) \alpha \\
& -\frac{1}{16}+\frac{7}{12}\left(k_{1}^{2}+k_{2}^{2}\right)-\frac{3}{4}\left(k_{1}^{4}+k_{2}^{4}\right)-\frac{13}{6} k_{1}^{2} k_{2}^{2} .
\end{aligned}
$$

\section{Third potential}

Here

$$
V_{3}=\frac{1}{2}\left[\frac{\alpha}{\left(\omega_{1}-\omega_{2}\right)^{2}}+\Omega^{2} \frac{1+4 \omega_{3}^{2}}{\left(\omega_{1}-\omega_{2}\right)^{4}}-\lambda \frac{\omega_{3}}{\left(\omega_{1}-\omega_{2}\right)^{3}}\right] .
$$

The corresponding Schrödinger equation admits separable solutions in two coordinate systems.

\section{Horicyclic coordinates}

$$
\omega_{1}=\frac{x^{2}+y^{2}+1}{2 y}, \quad \omega_{2}=\frac{x^{2}+y^{2}-1}{2 y}, \quad \omega_{3}=\frac{x}{y},
$$

where $y>0, x \in(-\infty, \infty)$.

\section{Semicircular parabolic coordinates}

$$
\omega_{1}=\frac{\left(\xi_{1}-\xi_{2}\right)^{2}+\frac{1}{4}}{2 \sqrt{-\xi_{1} \xi_{2}}}, \quad \omega_{2}=\frac{\left(\xi_{1}-\xi_{2}\right)^{2}-\frac{1}{4}}{2 \sqrt{-\xi_{1} \xi_{2}}}, \quad \omega_{3}=-\frac{\xi_{1}+\xi_{2}}{2 \sqrt{-\xi_{1} \xi_{2}}},
$$

where $\xi_{1}>0, \xi_{2}<0$.

In the case of horicyclic coordinates for the potential $V_{3}$, we get

$$
V_{3}(x, y)=\frac{1}{2}\left[\alpha y^{2}+\Omega^{2} y^{2}\left(y^{2}+4 x^{2}\right)-\lambda y^{2} x\right],
$$

and the Schrödinger equation assumes the form

$$
y^{2}\left(\frac{\partial^{2}}{\partial x^{2}}+\frac{\partial^{2}}{\partial y^{2}}\right) \Psi+\left[2 E-\alpha y^{2}-\Omega^{2}\left(y^{2}+4 x^{2}\right) y^{2}+\lambda x y^{2}\right] \Psi=0 .
$$

Putting $\Psi_{1}(x, y)=\psi_{1}(x) \psi_{2}(y)$, we have the separation equations in the form

$$
\begin{gathered}
\frac{d^{2} \psi_{1}}{d x^{2}}+\left(-\epsilon+\lambda x-4 \Omega^{2} x^{2}\right) \psi_{1}=0, \\
\frac{d^{2} \psi_{2}}{d y^{2}}+\left[(\epsilon-\alpha)-\Omega^{2} y^{2}-\frac{A^{2}-\frac{1}{4}}{y^{2}}\right] \psi_{2}=0,
\end{gathered}
$$

where $\epsilon$ is the horicyclic separation constant and $2 E=\frac{1}{4}-A^{2}$. Solving the first equation we have

$$
\psi_{1}(x) \equiv \psi_{n_{1}}=\left(\frac{2 \Omega}{\pi}\right)^{1 / 4} \frac{1}{\sqrt{2^{n_{1} n_{1} !}}} e^{-\Omega\left(x-\lambda / 8 \Omega^{2}\right)^{2}} H_{n_{1}}\left(\sqrt{2 \Omega}\left[x-\frac{\lambda}{8 \Omega^{2}}\right]\right),
$$

and the separation constant is 


$$
\epsilon=2 \Omega\left(2 n_{1}+1\right)-\frac{\lambda^{2}}{16 \Omega^{2}}
$$

For the second equation we get

$$
\psi_{2}(y) \equiv \psi_{n_{2}}(y)=\sqrt{\frac{2}{\Omega} \frac{n_{2} ! \Gamma(A+1)}{\Gamma(A) \Gamma\left(1+n_{2}+A\right)}}\left(\Omega y^{2}\right)^{A+1 / 2} e^{-(\Omega / 2) y^{2}} L_{n_{2}}^{A}\left(\Omega y^{2}\right),
$$

and

$$
A=\frac{\lambda^{2}}{32 \Omega^{3}}-\frac{\alpha}{2 \Omega}-2-2 N
$$

where $N=n_{1}+n_{2}$ is the principal quantum number. Here $H_{n}(x)$ is a Hermite polynomial and $L_{m}^{A}(z)$ a Laguerre polynomial. ${ }^{19,25}$ The discrete spectrum occurs for

$$
0 \leqslant N \leqslant \frac{1}{4 \Omega}\left(\frac{\lambda^{2}}{16 \Omega^{2}}-\alpha\right)
$$

The total bound state wave function $\Psi_{1}(x, y) \equiv \Psi_{n_{1} n_{2}}(x, y)$ is normalized by the condition

$$
\int_{-\infty}^{\infty} d x \int_{0}^{\infty} \frac{d y}{y^{2}} \Psi_{n_{1} n_{2}}(x, y) \Psi_{n_{1}^{\prime} n_{2}^{\prime}}(x, y)=\delta_{n_{1} n_{1}^{\prime}} \delta_{n_{2} n_{2}^{\prime}}
$$

The symmetry operator $L_{1}$ with eigenvalue $\epsilon$ is

$$
\begin{aligned}
L_{1} \Psi_{n_{1} n_{2}}(x, y) & \equiv\left[\left(K_{2}-M_{1}\right)^{2}-4 \Omega^{2} \frac{\omega_{3}^{2}}{\left(\omega_{1}-\omega_{2}\right)^{2}}+\lambda \frac{\omega_{3}}{\omega_{0}-\omega_{1}}\right] \Psi_{n_{1} n_{2}}(x, y) \\
& =\epsilon \Psi_{n_{1} n_{2}}(x, y)=\left[\frac{\lambda^{2}}{16 \Omega^{2}}-2 \Omega^{2}\left(2 n_{1}+1\right)\right] \Psi_{n_{1} n_{2}}(x, y) .
\end{aligned}
$$

Let us consider the semicircular parabolic coordinates. For the potential $V_{3}$ we obtain

$$
V_{3}\left(\xi_{1}, \xi_{2}\right)=-\xi_{1} \xi_{2}\left[8 \alpha+16 \lambda\left(\xi_{1}+\xi_{2}\right)+128 \Omega^{2}\left(\xi_{1}^{2}+\xi^{2}+\xi_{1} \xi_{2}\right)\right] .
$$

The Schrödinger equation in semicircular parabolic coordinates has the form

$$
\begin{aligned}
& \frac{2 \xi_{1} \xi_{2}}{\xi_{2}-\xi_{1}}\left[\left(\xi_{1} \frac{\partial^{2}}{\partial \xi_{1}^{2}}+\frac{1}{2} \frac{\partial}{\partial \xi_{1}}\right)-\left(\xi_{2} \frac{\partial^{2}}{\partial \xi_{2}^{2}}+\frac{1}{2} \frac{\partial}{\partial \xi_{2}}\right)\right] \Psi-\xi_{1} \xi_{2}\left[8 \alpha+16 \lambda\left(\xi_{2}+\xi_{1}\right)\right. \\
& \left.+128 \Omega^{2}\left(\xi_{1}^{2}+\xi_{2}^{2}+\xi_{2} \xi_{1}\right)\right] \Psi=E \Psi .
\end{aligned}
$$

Choosing the wave function as $\Psi_{2}=\psi_{1}\left(\xi_{1}\right) \psi_{2}\left(\xi_{2}\right)$ we obtain the two identical separation equations in the form

$$
\xi \frac{d^{2} \psi}{d \xi^{2}}+\frac{1}{2} \frac{d \psi}{d \xi}+\left(\mu+\frac{E}{\xi}-4 \alpha \xi-8 \lambda \xi^{2}-64 \Omega^{2} \xi^{3}\right) \psi=0,
$$

where $\psi=\psi_{1,2}, \xi=\xi_{1,2}$ and $\mu$ is the separation constant. The symmetry operator with eigenvalue $\mu$ is 


$$
\begin{aligned}
L_{2} \Psi_{2} \equiv & \frac{1}{\xi_{1}-\xi_{2}}\left[\xi_{2}\left(\xi_{2} \frac{\partial^{2}}{\partial \xi_{2}^{2}}+\frac{1}{2} \frac{\partial}{\partial \xi_{2}}\right)-\xi_{1}\left(\xi_{2} \frac{\partial^{2}}{\partial \xi_{2}^{2}}+\frac{1}{2} \frac{\partial}{\partial \xi_{2}}\right)\right] \Psi_{2} \\
& +\left[4 \alpha\left(\xi_{1}+\xi_{2}\right)+8 \lambda\left(\xi_{1} \xi_{2}+\xi_{1}^{2}+\xi_{2}^{2}\right)+64 \Omega^{2}\left(\xi_{1}+\xi_{2}\right)\left(\xi_{1}^{2}-\xi_{2}^{2}\right)\right] \Psi_{2} \\
\equiv & {\left[4\left\{K_{3}, K_{2}-M_{1}\right\}-2 \alpha \frac{\omega_{3}}{\omega_{1}-\omega_{2}}-\lambda \frac{4 \omega_{2}^{3}+1}{2\left(\omega_{1}-\omega_{2}\right)^{2}}-4 \Omega^{2} \frac{\omega_{3}\left(2 \omega_{3}^{2}+1\right)}{\left(\omega_{1}-\omega_{2}\right)^{3}}\right] \Psi_{2}=\mu \Psi_{2} . }
\end{aligned}
$$

The bound state solutions to these equations are of the form

$$
\Psi_{2}\left(\xi_{1}, \xi_{2}\right)=\exp \left[-4 \Omega\left(\xi_{2}^{2}+\xi_{1}^{2}\right)-\frac{\lambda}{2 \Omega}\left(\xi_{1}+\xi_{2}\right)\right]\left(\xi_{2} \xi_{1}\right)^{Q} \Pi_{j=1}^{N}\left(\xi_{1}-b_{j}\right)\left(\xi_{2}-b_{j}\right),
$$

where $Q=-\alpha / 4 \Omega+\lambda^{2} / 64 \Omega^{3}-N-\frac{3}{4}$. If we make this substitution, the zeros $b_{j}$ satisfy

$$
\sum_{j \neq l} \frac{b_{l}}{b_{l}-b_{j}}-2-4 N-\frac{\alpha}{\Omega}+\frac{\lambda^{2}}{16 \Omega^{3}}-\frac{2 \lambda}{\Omega} b_{l}-32 \Omega b_{l}^{2}=0,
$$

and the eigenvalues $\mu$ have the form

$$
\mu=-32 \Omega \sum_{l=1}^{N} b_{l}+\frac{\lambda}{2 \Omega}-\frac{\alpha \lambda}{\Omega^{2}}-\frac{\lambda^{3}}{32 \Omega^{4}} .
$$

The energy is quantized according to the condition $E=-Q(2 Q-1)$.

\section{Quadratic algebra relations}

$$
\begin{gathered}
{\left[L_{12}, L_{1}\right]=-8 \lambda L_{1}-16 \Omega^{2} L_{2}+8 \alpha \lambda,} \\
{\left[L_{12}, L_{2}\right]=96 L_{1}^{2}+128 \alpha L_{1}+8 L_{2}-256 \Omega^{2} H-96 \Omega^{2}+32 \alpha^{2},} \\
L_{12}^{2}=64 L_{1}^{3}+512 \Omega^{2} H L_{1}+8 \lambda\left\{L_{1}, L_{2}\right\}+128 \alpha L_{1}^{2}+16 \Omega^{2} L_{2}^{2} \\
+16 \alpha \lambda L_{2}+704 \Omega^{2} L_{1}+64 \alpha^{2} L_{1}-32 \lambda^{2} H-12 L^{2}+512 \alpha \Omega^{2},
\end{gathered}
$$

where $L_{12}=\left[L_{1}, L_{2}\right]$.

The quadratic algebra contains useful information in its relations. Indeed, if we consider the orthonormal bound state wave functions $\varphi_{m}$ and $\psi_{n}$ that satisfy $L_{1} \varphi_{m}=\lambda_{m} \varphi_{m}$ and $L_{2} \psi_{p}$ $=\rho_{p} \psi_{p}$, then the matrix with elements $D_{m n}$ where $L_{2} \varphi_{m}=\sum_{n=1}^{N} D_{m n} \varphi_{n}$ can readily be determined as follows. The quadratic algebra relations imply that

$$
\begin{gathered}
\left(\lambda_{m}-\lambda_{p}\right)^{2} D_{m p}=-16 \Omega^{2} D_{m p}+8 \lambda\left(\alpha-\lambda_{m}\right) \delta_{m p}, \\
\sum_{s=1}^{N} D_{s p} D_{m s}\left(\lambda_{p}+\lambda_{m}-2 \lambda_{s}\right)=8 D_{m p}+\left(96 \lambda_{m}^{2}+128 \alpha \lambda_{m}-256 \Omega^{2} E+96 \Omega^{2}+32 \alpha^{2}\right) \delta_{m p},
\end{gathered}
$$

where $\lambda_{n}=2 \Omega(2 n+1)-\left(\lambda^{2} / 16 \Omega^{2}\right)$. The first of these conditions implies that the only nonzero components of $D_{p q}$ are $D_{m, m+1}, D_{m+1, m}$, and $D_{m m}=8 \lambda\left(\alpha-\lambda_{m}\right)$. The second relation implies

$$
\Omega\left[D_{p-1, p}^{2}-D_{p+1, p}^{2}\right]=12 \lambda_{p}^{2}+16 \alpha \lambda_{p}-32 \Omega^{2} E+12 \Omega^{2}+4 \alpha^{2}+8 \lambda \alpha-8 \lambda \lambda_{p} .
$$


There is no further information available about the matrix elements $D_{p q}$. Indeed, the matrix elements are essentially determined by these relations. We could also consider the matrix $C$ with elements defined by

$$
L_{1} \psi_{p}=\sum_{k=1}^{N} C_{p k} \psi_{k}
$$

The quadratic algebra implies that $C$ satisfies certain relations, such as

$$
C_{n p}\left(\rho_{p}-\rho_{n}\right)^{2}=96 \sum_{m=1}^{N} C_{m p} C_{n m}+128 \alpha C_{n p}+\left(\rho_{n}-256 \Omega^{2} E+96 \Omega^{2}+32 \alpha^{2}\right) \delta_{n p} .
$$

One interesting condition we deduce from this is that

$$
96 \operatorname{Tr}\left(C^{2}\right)+128 \alpha \operatorname{Tr}(C)+\sum_{n=1}^{N} \rho_{n}+N\left(32 \alpha^{2}-256 \Omega^{2} E+96 \Omega^{2}\right)=0,
$$

where $\operatorname{Tr}$ is the trace function. We can also introduce the matrices that relate the various bases that we are considering via $\varphi_{m}=\sum_{s=1}^{N} A_{m s} \psi_{s}$ and $\psi_{m}=\sum_{s=1}^{N} B_{m s} \varphi_{s}$. We note, in particular, that

$$
\sum_{p=1}^{N} D_{m p} A_{p s}=\rho_{s} A_{m s}
$$

i.e., the vector $v^{(s)}=\left(A_{1 s}, \ldots, A_{N s}\right)$ is an eigenvector of $D$ with eigenvalues $\rho_{s}$. In fact, the eigenvalues of $D$ are solutions of the characteristic equation written in continued fraction form,

$$
\left.\left.\left.\frac{\beta_{N}}{\alpha_{N}} \gamma_{N-1}=\beta_{N-1}-\alpha_{N-1} \gamma_{N-2}\right] \beta_{N-2}-\alpha_{N-2} \gamma_{N-3}\right] \cdots\right] \beta_{2}-\alpha_{2} \gamma_{1} / \beta_{1},
$$

where $\alpha_{n}=D_{n n-1}, \beta_{n}=D_{n n}-\rho$, and $\gamma_{n}=D_{n n+1}$.

\section{A DIRECT INTERBASIS EXPANSION}

As an example of a direct interbasis expansion we consider the singular oscillator (4). For a fixed value of $E_{N}$, we can write the equidistant wave function (17) in terms of the spherical wave function (7) as

$$
\Psi_{n_{1} n_{2}}=\sum_{m=0}^{n_{1}+n_{2}} W_{n_{1} n_{2}}^{n m}\left(k_{1}, \pm k_{2}, \pm k_{3}\right) \Psi_{n m}
$$

where $n_{1}+n_{2}=n+m$. The connection between the equidistant $(a, b)$ and spherical $(\tau, \varphi)$ coordinates is

$$
\sinh a=\sinh \tau, \quad \sinh b=\frac{\tanh \tau \cos \varphi}{\sqrt{1-\tanh ^{2} \tau \cos ^{2} \varphi}}
$$

Using spherical coordinates on the left side of expansion (70), then, considering the limit $\tau \rightarrow \infty$ in the so-obtained equation and the formula

$$
\lim _{x \rightarrow 1}{ }_{2} F_{1}(-n, \alpha ; \beta ; x) \rightarrow \frac{\Gamma(\beta) \Gamma(\beta-\alpha+n)}{\Gamma(\beta-\alpha) \Gamma(\beta+n)},
$$


we see that dependence on $\tau$ cancels on both sides of (70). Now using the orthogonality condition of the angular wave function (10), we find the following expression for the interbasis coefficients $W_{n_{1} n_{2}}^{n m}$ :

$$
W_{n_{1} n_{2}}^{n m}=(-1)^{m} \sqrt{\frac{\Gamma\left(k_{1}-n\right) \Gamma\left(1+m \pm k_{2} \pm k_{3}\right) m ! n_{1} !}{\Gamma\left(1+m \pm k_{2}\right) \Gamma\left(1+m \pm k_{3}\right) n_{2} ! n !}} A_{n_{1} n_{2}}^{n m} B_{n_{1} n_{2}}^{n m},
$$

where

$$
\begin{aligned}
A_{n_{1} n_{2}}^{n m}= & \sqrt{\left(k_{1} \mp k_{2}-1-2 n_{1}\right)\left(2 m \pm k_{2} \pm k_{3}+1\right) \Gamma\left(k_{1} \mp k_{2}-1-n_{2}-2 n_{1}\right)} \\
& \times \sqrt{\frac{\Gamma\left(k_{1}-n_{1}\right) \Gamma\left(k_{1} \mp k_{2} \mp k_{3}-1-n_{2}-2 n_{1}\right) \Gamma\left(k_{1} \mp k_{2} \mp k_{3}-1-n-2 m\right)}{\Gamma\left(k_{1} \pm k_{3}+n_{2}\right) \Gamma\left(k_{1} \mp k_{2}-n_{1}\right) \Gamma\left(k_{1} \pm k_{2}+n_{1}\right) \Gamma\left(2+n+2 m \pm k_{2} \pm k_{3}\right)}}
\end{aligned}
$$

and

$$
B_{n_{1} n_{2}}^{n m}=\frac{1}{2} \int_{0}^{\pi / 2}(\sin \varphi)^{1+2 n_{2} \pm k_{3}}(\cos \varphi)^{1 \pm k_{2}} P_{n_{1}}^{\left(k_{1} \pm k_{2}-1-2 n_{1}, \pm k_{2}\right)}(\cos 2 \varphi) P_{m}^{\left( \pm k_{3}, \pm k_{2}\right)}(\cos 2 \varphi) d \varphi
$$

The integral $B_{n_{1}, n_{2}}^{n m}$ can be evaluated by means of ${ }^{25}$

$$
\begin{aligned}
\int_{-1}^{1}(1-x)^{\tau}(1+x)^{\beta} P_{n}^{(\alpha, \beta)}(x) P_{m}^{(\rho, \beta)}(x) d x \\
=\frac{2^{\beta+\tau+1} \Gamma(\alpha-\tau+n) \Gamma(\beta+1+n)}{m ! n ! \Gamma(1+\rho) \Gamma(\alpha-\tau)} \frac{\Gamma(1+m+\rho) \Gamma(1+\tau)}{\Gamma(2+n+\beta+\tau)} \\
\quad \times_{4} F_{3}\left(\begin{array}{c}
-m, 1+m+\beta+\rho, 1+\tau, 1+\tau-\alpha \\
1+\rho, 2+n+\beta+\tau, 1-n+\tau-\alpha
\end{array} \mid 1\right) .
\end{aligned}
$$

We thus obtain

$$
\begin{aligned}
W_{n_{1} n_{2}}^{n m}= & \frac{(-1)^{m} \Gamma\left(k_{1} \mp k_{2} \mp k_{3}-1-n-m\right)}{\Gamma\left(2+n+m \pm k_{2} \pm k_{3}\right) \Gamma\left(1 \pm k_{3}\right)} \sqrt{\frac{\left(k_{1} \mp k_{2}-1-2 n_{1}\right)\left(2 m \pm k_{2} \pm k_{3}+1\right)}{\Gamma\left(k_{1} \mp k_{2} \mp k_{3}-1-n_{2}-2 n_{1}\right)}} \\
& \times \sqrt{\Gamma\left(1+n_{1} \pm k_{2}\right) \Gamma\left(k_{1}-n_{1}\right) \Gamma\left(1+n_{2} \pm k_{3}\right) \Gamma\left(k_{1} \mp k_{2} \mp k_{3}-1-n-2 m\right)} \\
& \times \sqrt{\frac{\Gamma\left(k_{1} \mp k_{2}-1-n_{2}-2 n_{1}\right) \Gamma\left(1+m \pm k_{2} \pm k_{3}\right) \Gamma\left(1+m \pm k_{3}\right) \Gamma\left(k_{1}-n\right)}{\Gamma\left(k_{1} \mp k_{2}-n_{1}\right) \Gamma\left(2+n_{1}+n_{2}+m \pm k_{2} \pm k_{3}\right) \Gamma\left(1+m \pm k_{2}\right) n_{1} ! n_{2} ! n ! m !}} \\
& \times{ }_{4} F_{3}\left(\begin{array}{c}
-m, 1+m \pm k_{2} \pm k_{3}, 1+n_{2} \pm k_{3}, 2+n_{2}+2 n_{1} \pm k_{2} \pm k_{3}-k_{1} \\
1 \pm k_{3}, 2+n_{1}+n_{2} \pm k_{2} \pm k_{3}, 2+n_{1}+n_{2} \pm k_{2} \pm k_{3}-k_{1}
\end{array} \mid 1\right),
\end{aligned}
$$

a closed form expression for the interbasis expansion coefficients. Note that the hypergeometric function ${ }_{4} F_{3}$ is of the Saalschutzian type. ${ }^{19,26}$

\section{ACKNOWLEDGMENTS}

The authors thank P. Winternitz for very interesting discussions.

Work supported in part by the National Science Foundation under Grant No. DMS 94-00533

${ }^{1}$ E. G. Kalnins, W. Miller, Jr., and G. S. Pogosyan, “Superintegrability and associated polynomial solutions. Euclidean space and the sphere in two dimensions," J. Math. Phys. 37, 6439 (1996). 
${ }^{2}$ N. W. Evans, "Superintegrability in classical mechanics," Phys. Rev. A 41, 5666 (1990); "Group theory of the Smorodinsky-Winternitz system," J. Math. Phys. 32, 3369 (1991).

${ }^{3}$ S. Wojciechovski, "Superintegrability of the Calogero-Moser system," Phys. Lett. A 95, 279 (1983).

${ }^{4}$ N. W. Evans, "Super-integrability of the Winternitz system," Phys. Lett. A 147, 483 (1990).

${ }^{5}$ C. P. Boyer, E. G. Kalnins, and P. Winternitz, "Completely integrable relativistic Hamiltonian systems and separation of variables in Hermitian hyperbolic spaces," J. Math. Phys. 24, 2022 (1983).

${ }^{6}$ C. Grosche, G. S. Pogosyan, and A. N. Sissakian, "Path integral approach to superintegrable potentials. The twodimensional hyperboloid," Phys. Part. Nucl. 27, 244 (1996).

${ }^{7}$ L. P. Eisenhart, "Enumeration of potentials for which one-particle Schrödinger equations are separable," Phys. Rev. 74, 87 (1948).

${ }^{8}$ J. Frǐ̌, V. Mandrosov, Ya. A. Smorodinsky, M. Uhlir, and P. Winternitz, "'On higher symmetries in quantum mechanics," Phys. Lett. 16, 354 (1965).

${ }^{9}$ M. N. Olevskii, "Triorthogonal systems in spaces of constant curvature in which the equation $\Delta_{2} u+\lambda u=0$ allows the complete separation of variables," Math. Sb. 27, 379 (1950) (in Russian).

${ }^{10}$ J. Frǐ̌, Ya. A. Smorodinskii, M. Uhlír, and P. Winternitz, “ Symmetry groups in classical and quantum mechanics," Sov. J. Nucl. Phys. 4, 444 (1967).

${ }^{11}$ Ya. A. Granovsky, A. S. Zhedanov, and I. M. Lutzenko, "Quadratic algebra as a 'hidden' symmetry of the Hartmann potential,", J. Phys. A 24, 3887 (1991).

${ }^{12}$ C. Grosche, G. S. Pogosyan, and A. N. Sissakian, "Path integral discussion for Smorodinsky-Winternitz potentials: I. Two- and three dimensional Euclidean space," Fortschr. Phys. 43, 453 (1995).

${ }^{13}$ E. G. Kalnins, W. Miller, and M. Tratnik, "Families of orthogonal and biorthogonal polynomials on the $n$-sphere," SIAM J. Math. Anal. 22, 272 (1991).

${ }^{14}$ E. G. Kalnins and W. Miller, "Hypergeometric expansions of Heun polynomials," SIAM J. Math. Anal. 22, 1450 (1991).

${ }^{15}$ E. G. Kalnins and W. Miller, "Orthogonal polynomials on $n$-spheres: Gegenbauer, Jacobi and Heun," in Topics in Polynomials of One and Several Variables and their Applications: A Legacy of P. L. Chebyshev (1821-1894), edited by H. M. Srivastava, T. M. Rassias, and A. Yanushauskas (World Scientific, Singapore, 1991).

${ }^{16}$ J. Patera and P. Winternitz, "A new basis for the representation of the rotation group. Lamé and Heun Polynomials," J. Math. Phys. 14, 1130 (1973).

${ }^{17}$ H. L. Krall and I. M. Sheffer, “Orthogonal polynomials in two variables," Ann. Mat. Pure Appl. 76, 325 (1967).

${ }^{18}$ W. Miller, Jr., Symmetry and Separation of Variables (Addison-Wesley, Providence, RI, 1977).

${ }^{19}$ A. Erdélyi, W. Magnus, F. Oberhettinger, and F. G. Tricomi (editors), Higher Transcendental Functions (McGraw-Hill, New York, 1953), Vols. I, II.

${ }^{20}$ A. Frank and K. B. Wolf, "Lie algebras for systems with mixed spectra. I. The scattering Pöschl-Teller potential," J. Math. Phys. 25, 973 (1985).

${ }^{21}$ A. O. Barut, A. Inomata, and R. Wilson, “Algebraic treatment of the second Pöschl-Teller, Morse-Rosen and Eckart equations," J. Phys. A. 20, 4075 (1987).

${ }^{22}$ E. G. Kalnins and W. Miller, "Separable coordinates, integrability and the Niven equations," J. Phys. A 25, 5663 (1992).

${ }^{23}$ M. F. Manning and N. Rosen, "Potential function for vibrations of diatomic molecules," Phys. Rev. 44, 953 (1933).

${ }^{24}$ P. W. Higgs, "Dynamical symmetries in a spherical geometry," J. Phys. A 12, 309 (1979).

${ }^{25}$ A. P. Prudnikov, Yu. A. Brychkov, and O. I. Marichev, Integrals and Series, Special Functions (Nauka, Moscow, 1986).

${ }^{26}$ J. A. Wilson, "Some hypergeometric orthogonal polynomials," SIAM J. Math. Anal. 11, 690 (1980). 